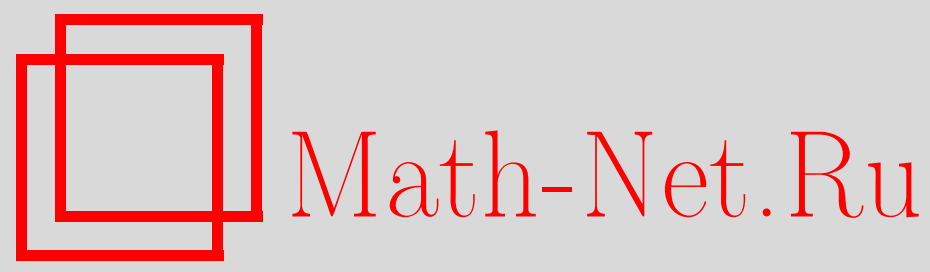

Г. А. Рудых, Э. И. Семенов, Неавтомодельные решения многомерного уравнения нелинейной диффузии, Матем. заметки, 2000, том 67, выпуск 2, 250-256

DOI: https://doi.org/10.4213/mzm833

Использование Общероссийского математического портала Math-Net.Ru подразумевает, что вы прочитали и согласны с пользовательским соглашением http://www . mathnet.ru/rus/agreement

Параметры загрузки:

IP: 34.239 .49 .27

26 апреля 2023 г., 13:47:44 


\title{
НЕАВТОМОДЕЛЬНЫЕ РЕШЕНИЯ МНОГОМЕРНОГО УРАВНЕНИЯ НЕЛИНЕЙНОЙ ДИФФУЗИИ
}

\author{
Г. А. Рудых, Э.И. Семёнов
}

\begin{abstract}
Предлагается и исследуется оригинальная конструкция точного неотрицательного решения многомерного уравнения нелинейной диффузии. После подстановки предъявленной конструкции в исходное уравнение приходим к исследованию системы алгебро-дифференциальных уравнений, в которой число уравнений превосходит число искомых функций. Доказано, что полученная система имеет решения отличные от тривиалього. На основе этого результата построены точные неавтомодельные анизотропные по пространственным переменным явные неотрицательные решения как класса уравнений пористой среды (нестационарной фильтрации), так и класса уравнений с отрицательньм показателем в коэффициенте нелинейной теплопроводности. В частности, в этот класс вкладываются так называемые уравнения быстрой и предельной диффузии.

Библиография: 23 названия.
\end{abstract}

1. Настоящая работа примыкает к исследованиям [1]-[13] и посвящена построению точных неавтомодельных анизотропных по пространственным переменным явных неотрицательных решений многомерного уравнения нелинейной диффузии

$$
u_{t}=\nabla \cdot\left(u^{\lambda} \nabla u\right), \quad u=u(\boldsymbol{x}, t): \Omega \times \overline{\mathbb{R}}^{+} \rightarrow \mathbb{R}^{+}, \quad \boldsymbol{x} \in \mathbb{R}^{n},
$$

где $\Omega \subset \mathbb{R}^{n}$ - область; $\mathbb{R}^{+}=(0, \infty) ; u(\boldsymbol{x}, t) \geqslant 0$ - температура среды; $\lambda \in \mathbb{R}$ - параметр нелинейной среды, значения которого различны для различных процессов переноса тепла. Уравнение (1) принадлежит классу так назьваемых неявно вырождающихся параболических уравнений [14]. Ниже предлагается и исследуется оригинальная конструкция [15]-[17] точного неотрицательного решения уравнения (1) в виде “конечной суммы" (см. работу [18] и приведенные в ней ссылки). В итоге после подстановки предъявленной конструкции в уравнение (1) приходим к исследованию системы алгебро-дифференциальных уравнений (АДУ), которая является переопределенной (число уравнений превосходит число искомых функций). Известно [19], что переопределенные системы уравнений могут вообще не иметь решений. Доказано, что полученная система (АДУ) имеет решения отличные от тривиального. На основе этого результата показано, что введенная конструкция позволяет получить точные неотрицательные решения как класса уравнений пористой среды (нестационарной фильтрации), когда $\lambda>0$, так и класса уравнений (1) с отрицательным показателем $\lambda$ в коэффициенте нелинейной теплопроводности $K(u)=u^{\lambda}$. В частности, в этот класс вкладьваются так назьваемые 
уравнения быстрой $(-1<\lambda<0)$ и предельной $(\lambda=-1)$ диффузии. Отметим, что в основном полученные в этой работе точные неотрицательные решения отмеченных вьше уравнений не являются инвариантными с точки зрения групп точечных преобразований и групп Ли-Беклунда [20], [21].

2. Введем в рассмотрение функции

$$
Z_{k}(\boldsymbol{x}, t)=\frac{1}{2}\left(\boldsymbol{x}, A_{k}(t) \boldsymbol{x}\right)+\left(\boldsymbol{x}, \boldsymbol{B}_{k}(t)\right)+C_{k}(t),
$$

где $\boldsymbol{x} \in \mathbb{R}^{n} ; A_{k}(t)=\left[a_{k i j}(t)\right]$ - вещественные симметричные $n \times n$-матрицы; $\boldsymbol{B}_{k}(t)=$ $\left(b_{k 1}(t), \ldots, b_{k n}(t)\right)^{\prime}$ - вектор-столбцы; $C_{k}(t)$ - скалярные функции; $a_{k i j}(t), b_{k i}(t), C_{k}(t)$ $\in C^{1}\left(\overline{\mathbb{R}}^{+}\right)$- вещественные функции; $(\cdot, \cdot)$ - скалярное произведение в $\mathbb{R}^{n} ; k=1,2$, $i, j=1,2, \ldots, n$.

Теорема 1. Уравнение (1) имеет точное неотрицательное решение вида

$$
u(\boldsymbol{x}, t)=\left[\lambda\left[Z_{1}(\boldsymbol{x}, t)\right]_{+}^{p}+\lambda Z_{2}(\boldsymbol{x}, t)\right]_{+}^{1 / \lambda},
$$

если функции $A_{k}(t), \boldsymbol{B}_{k}(t), C_{k}(t)$ связаны соотношениями

$$
\begin{aligned}
\frac{\partial}{\partial t} Z_{2} & =\lambda Z_{2} \Delta Z_{2}+\left|\nabla Z_{2}\right|^{2}, \quad \lambda Z_{1} \Delta Z_{1}+[p(\lambda+1)-\lambda]\left|\nabla Z_{1}\right|^{2}=0, \\
p Z_{1} \frac{\partial}{\partial t} Z_{1} & =p \lambda Z_{1} Z_{2} \Delta Z_{1}+\lambda Z_{1}^{2} \Delta Z_{2}+\lambda p(p-1) Z_{2}\left|\nabla Z_{1}\right|^{2}+2 p Z_{1}\left(\nabla Z_{1}, \nabla Z_{2}\right),
\end{aligned}
$$

əде $[\cdot]_{+}=\max \{[\cdot], 0\}, \lambda, p \in \mathbb{R} \backslash\{0\}$.

Пусть $\xi=p(\lambda+1)-\lambda, \xi \neq 0$. Тогда система уравнений (4) запишется в виде

$$
\begin{gathered}
\frac{\partial}{\partial t} Z_{2}=\lambda Z_{2} \Delta Z_{2}+\left|\nabla Z_{2}\right|^{2}, \quad \lambda Z_{1} \Delta Z_{1}+\xi\left|\nabla Z_{1}\right|^{2}=0 \\
\frac{\partial}{\partial t} Z_{1}=\sigma Z_{2} \Delta Z_{1}+\tau Z_{1} \Delta Z_{2}+2\left(\nabla Z_{1}, \nabla Z_{2}\right),
\end{gathered}
$$

где $\sigma=p \lambda / \xi, \tau=\lambda / p, \lambda, p \in \mathbb{R} \backslash\{0\}$.

Теорема 2. Пусть $A_{k}(t)$ - вещественные симметричные матрицы с әлементами $a_{k i j}(t) \in C^{1}\left(\overline{\mathbb{R}}^{+}\right), \boldsymbol{B}_{k}(t)$ - вектор-столбиь с компонентами $b_{k i}(t) \in C^{1}\left(\overline{\mathbb{R}}^{+}\right)$ и $C_{k}(t) \in C^{1}\left(\overline{\mathbb{R}}^{+}\right)$- скалярные функиии. Тогда, для того чтобы функиии $Z_{1}, Z_{2}$, определяемые соотношением (2), удовлетворяли системе уравнений (5), необходимо и достаточно, чтобъ $A_{k}(t), \boldsymbol{B}_{k}(t), C_{k}(t)$ удовлетворяли системе АДУ

$$
\begin{gathered}
\dot{A}_{2}=2 A_{2}^{2}+\lambda\left(\operatorname{tr} A_{2}\right) A_{2}, \quad \dot{\boldsymbol{B}}_{2}=2 A_{2} \boldsymbol{B}_{2}+\lambda\left(\operatorname{tr} A_{2}\right) \boldsymbol{B}_{2}, \\
\dot{C}_{2}=\left|\boldsymbol{B}_{2}\right|^{2}+\lambda\left(\operatorname{tr} A_{2}\right) C_{2}, \\
\dot{A}_{1}=4 A_{1} A_{2}+\tau\left(\operatorname{tr} A_{2}\right) A_{1}+\sigma\left(\operatorname{tr} A_{1}\right) A_{2}, \\
\dot{\boldsymbol{B}}_{1}=2\left(A_{1} \boldsymbol{B}_{2}+A_{2} \boldsymbol{B}_{1}\right)+\tau\left(\operatorname{tr} A_{2}\right) \boldsymbol{B}_{1}+\sigma\left(\operatorname{tr} A_{1}\right) \boldsymbol{B}_{2}, \\
\dot{C}_{1}=2\left(\boldsymbol{B}_{1}, \boldsymbol{B}_{2}\right)+\tau\left(\operatorname{tr} A_{2}\right) C_{1}+\sigma\left(\operatorname{tr} A_{1}\right) C_{2}, \\
\lambda\left(\operatorname{tr} A_{1}\right) A_{1}+2 \xi A_{1}^{2}=0, \quad \lambda\left(\operatorname{tr} A_{1}\right) \boldsymbol{B}_{1}+2 \xi A_{1} \boldsymbol{B}_{1}=0, \quad \lambda\left(\operatorname{tr} A_{1}\right) C_{1}+\xi\left|\boldsymbol{B}_{1}\right|^{2}=0,(8)
\end{gathered}
$$
әде $\operatorname{tr} A_{k}=\sum_{i=1}^{n} a_{k i i}(t)-$ след матрицьь $A_{k}(t), k=1,2$.

Теоремы 1, 2 приводят к следующему результату. 
УТВЕРЖДЕНИЕ 1. Если вещественные симметричные матрицы $A_{k}(t)$ с әлементами $a_{k i j}(t) \in C^{1}\left(\overline{\mathbb{R}}^{+}\right)$, вектор-столби, $\boldsymbol{B}_{k}(t)$ с компонентами $b_{k i}(t) \in C^{1}\left(\overline{\mathbb{R}}^{+}\right)$ и скалярные функиии $C_{k}(t) \in C^{1}\left(\overline{\mathbb{R}}^{+}\right)$удовлетворяют переопределенной системе уравнений (6)-(8), то функция (3) является точным неотрицательным решением уравнения (1).

Если в $(3)$ положить $Z_{1}(\boldsymbol{x}, t) \equiv 0$, то $A_{1}(t) \equiv 0, \boldsymbol{B}_{1}(t) \equiv 0, C_{1}(t) \equiv 0$ и

$$
u(\boldsymbol{x}, t)=\left[\lambda\left[\frac{1}{2}\left(\boldsymbol{x}, A_{2}(t) \boldsymbol{x}\right)+\left(\boldsymbol{x}, \boldsymbol{B}_{2}(t)\right)+C_{2}(t)\right]\right]_{+}^{1 / \lambda}
$$

В этом случае система АДУ (6)-(8) сводится к системе обыкновенных дифференциальных уравнений (ОДУ) (6) и справедлива

ТЕОРемА 3. Пусть заданы вещественная симметричная матрица $A_{2}(0) \in$ $M_{n}(\mathbb{R})$, вектор-столбеи, $\boldsymbol{B}_{2}(0) \in M_{n, 1}(\mathbb{R})$ и скаляр $C_{2}(0) \in \mathbb{R}$. Пусть, помимо этого, $z(t)$ - вещественное решение задачи Коши

$$
\dot{z}(t)=\prod_{l=1}^{n}\left[1-2 d_{l}(0) z(t)\right]^{-\lambda / 2}, \quad z(0)=0, \quad \dot{z}(t)=\frac{d}{d t} z(t) .
$$

Тогда вещественное решение задачи Коши для системы ОДУ

$$
\begin{array}{cc}
\dot{A}_{2}(t)=2 A_{2}^{2}(t)+\lambda\left[\operatorname{tr} A_{2}(t)\right] A_{2}(t), & \left.A_{2}(t)\right|_{t=0}=A_{2}(0), \\
\dot{B}_{2}(t)=2 A_{2}(t) \boldsymbol{B}_{2}(t)+\lambda\left[\operatorname{tr} A_{2}(t)\right] \boldsymbol{B}_{2}(t), & \left.\boldsymbol{B}_{2}(t)\right|_{t=0}=\boldsymbol{B}_{2}(0), \\
\dot{C}_{2}(t)=\left|\boldsymbol{B}_{2}(t)\right|^{2}+\lambda\left[\operatorname{tr} A_{2}(t)\right] C_{2}(t), & \left.C_{2}(t)\right|_{t=0}=C_{2}(0),
\end{array}
$$

имеет вид

$$
\begin{aligned}
A_{2}(t)=\dot{z}(t) S Q(t) D(0) S^{\prime} & =\dot{z}(t) S Q(t) S^{\prime} A_{2}(0), \\
\boldsymbol{B}_{2}(t)=\dot{z}(t) S Q(t) S^{\prime} \boldsymbol{B}_{2}(0), \quad C_{2}(t) & =\dot{z}(t) C_{2}(0)+z(t)\left(\boldsymbol{B}_{2}(0), \boldsymbol{B}_{2}(t)\right) .
\end{aligned}
$$

Причем $A_{2}(t)$ - симметричная матрича для всех $t \in \operatorname{domain} A_{2}(t)$, где $S \in M_{n}(\mathbb{R})$ вещ,ственная ортогональная матрица, $D(0)=\operatorname{diag}\left[d_{1}(0), \ldots, d_{n}(0)\right]$,

$$
Q(t)=\operatorname{diag}\left[\left[1-2 d_{1}(0) z(t)\right]^{-1}, \ldots,\left[1-2 d_{n}(0) z(t)\right]^{-1}\right], \quad A_{2}(0)=S D(0) S^{\prime},
$$

$d_{l}(0) \in \mathbb{R} \backslash\{0\}-$ собственные значения матрицы $A_{2}(0) ; \lambda \in \mathbb{R} \backslash\{0\}$.

Из утверждения 1 и теоремы 3 вытекает

СлЕДСТВИЕ 1. Уравнение (1) обладает точным неавтомодельным анизотропным по пространственным переменным явным неотрицательным решением (9). $\Pi$ Пи этом $A_{2}(t), \boldsymbol{B}_{2}(t), C_{2}(t)$ определяются согласно (12).

3. Перейдем к исследованию системы АДУ (6)-(8). 
УТвеРЖДЕнИЕ 2. Пусть $A_{1}(t)=\varphi(t) S E_{m} S^{\prime} \neq 0, E_{m}=\operatorname{diag}\left[e_{1}, \ldots, e_{n}\right], e_{k} \in\{0,1\}$, $k=1,2, \ldots, n, \operatorname{rank} E_{m}=m \in\{1,2, \ldots, n\}, \varphi(t)-$ произвольная вещественная функиия, обладающая тем свойством, что $\varphi(t) \neq 0$ для всех $t \in \operatorname{domain} A_{1}(t)$, $S \in M_{n}(\mathbb{R})$ - вещественная ортогональная матрица. Тогда если $m=-2 \xi / \lambda$, то $A_{1}(t)$ является решением матричного уравнения системы (8) и выполняется соотношение $\operatorname{rank} E_{m}=\operatorname{rank} A_{1}(t)=-2 \xi / \lambda$, әде $\xi=p(\lambda+1)-\lambda, \xi \neq 0, \lambda, p \in \mathbb{R} \backslash\{0\}$.

Тем самым, в силу зависимости $m=-2 \xi / \lambda$ и с учетом вида матрицы $A_{1}(t)$ векторное и скалярное уравнения системы (8) соответственно запишутся в виде

$$
\left(I-E_{m}\right) S^{\prime} \boldsymbol{B}_{1}(t)=0, \quad\left|\boldsymbol{B}_{1}(t)\right|^{2}=2 \varphi(t) C_{1}(t) .
$$

Teорема 4. Eсли $p=1 / 2, \lambda=-1 /(m-1), m \in\{2, \ldots, n\}, m o \xi=m /(2(m-1))$, $\tau=-2 /(m-1), \sigma=-1 / m$ и система АДУ (6)-(8) обладает решением

$$
\begin{gathered}
A_{1}(t)=\beta[h(t)]_{+}^{-(m-3) /(m-2)} S E_{m} S^{\prime}, \quad A_{2}(t)=\alpha[h(t)]^{-1} S E_{m} S^{\prime}, \\
\boldsymbol{B}_{1}(t)=\left[\boldsymbol{B}_{1}(0)-\frac{\beta}{\alpha} \boldsymbol{B}_{2}(0)\right][h(t)]_{+}^{2 /(m-2)}+\frac{\beta}{\alpha} \boldsymbol{B}_{2}(0)[h(t)]_{+}^{-(m-3) /(m-2)}, \\
\boldsymbol{B}_{2}(t)=[h(t)]^{-1} \boldsymbol{B}_{2}(0), \\
C_{1}(t)=\frac{1}{2 \beta}\left|\boldsymbol{B}_{1}(0)-\frac{\beta}{\alpha} \boldsymbol{B}_{2}(0)\right|^{2}[h(t)]_{+}^{(m+1) /(m-2)} \\
+\frac{1}{\alpha}\left[\left(\boldsymbol{B}_{1}(0), \boldsymbol{B}_{2}(0)\right)-\frac{\beta}{\alpha}\left|\boldsymbol{B}_{2}(0)\right|^{2}\right][h(t)]_{+}^{2 /(m-2)}+\frac{\beta}{2 \alpha^{2}}\left|\boldsymbol{B}_{2}(0)\right|^{2}[h(t)]_{+}^{-(m-3) /(m-2)}, \\
C_{2}(t)=-\frac{\alpha}{2 \beta^{2}}\left|\boldsymbol{B}_{1}(0)-\frac{\beta}{\alpha} \boldsymbol{B}_{2}(0)\right|^{2}[h(t)]_{+}^{m /(m-2)}+\frac{1}{2 \alpha}\left|\boldsymbol{B}_{2}(0)\right|^{2}[h(t)]^{-1},
\end{gathered}
$$

причем $\left(I-E_{m}\right) S^{\prime} \boldsymbol{B}_{1}(0)=\left(I-E_{m}\right) S^{\prime} \boldsymbol{B}_{2}(0)=0$, где $h(t)=1-\alpha t(m-2) /(m-1)$, $\boldsymbol{B}_{1}(0), \boldsymbol{B}_{2}(0) \in \mathbb{R}^{n}$ - постоянные векторы, $\alpha, \beta \in \mathbb{R} \backslash\{0\}$.

Отметим, что фактическим следствием утверждения 1 и теоремы 4 является следуюший результат.

Теорема 5. Уравнение нелинейной диффузии

$$
u_{t}=\nabla \cdot\left(u^{-1 /(m-1)} \nabla u\right), \quad u \triangleq u(x, t): \Omega \times \overline{\mathbb{R}}^{+} \rightarrow \mathbb{R}^{+}, \quad \boldsymbol{x} \in \mathbb{R}^{n},
$$

обладает точным неавтомодельным анизотропным по пространственныц переменным явным неотрицательным решением вида (3) при р $=1 / 2$ и $\lambda=-1 /(m-1)$. При этом функиии $A_{k}(t), \boldsymbol{B}_{k}(t), C_{k}(t)$ определяются формулами (13), где $k=1,2$, $m \in\{2, \ldots, n\}$.

Если $m=2$, то из теоремы 4 вытекает важное, как нам представляется,

СледСтвие 2. Если $p=1 / 2, \lambda=-1, m=2, \operatorname{mo} \tau=-2, \sigma=-1 / 2, \xi=1 u$ система АДУ (6)-(8) имеет следующее решение:

$$
\begin{gathered}
A_{1}(t)=\beta e^{-\alpha t} S E_{2} S^{\prime}, \quad A_{2}(t)=\alpha S E_{2} S^{\prime}, \\
\boldsymbol{B}_{1}(t)=e^{-2 \alpha t}\left[\frac{\beta}{\alpha}\left(e^{\alpha t}-1\right) \boldsymbol{B}_{2}(0)+\boldsymbol{B}_{1}(0)\right], \quad \boldsymbol{B}_{2}(t)=\boldsymbol{B}_{2}(0), \\
C_{1}(t)=\frac{1}{2 \beta} e^{-3 \alpha t}\left|\frac{\beta}{\alpha}\left(e^{\alpha t}-1\right) \boldsymbol{B}_{2}(0)+\boldsymbol{B}_{1}(0)\right|^{2}, \\
C_{2}(t)=-\frac{\alpha}{2 \beta^{2}} e^{-2 \alpha t}\left|\frac{\beta}{\alpha} \boldsymbol{B}_{2}(0)-\boldsymbol{B}_{1}(0)\right|^{2}+\frac{1}{2 \alpha}\left|\boldsymbol{B}_{2}(0)\right|^{2},
\end{gathered}
$$


причем $\left(I-E_{2}\right) S^{\prime} \boldsymbol{B}_{1}(0)=\left(I-E_{2}\right) S^{\prime} \boldsymbol{B}_{2}(0)=0$, где $\boldsymbol{B}_{1}(0), \boldsymbol{B}_{2}(0) \in \mathbb{R}^{n}, \alpha, \beta \in \mathbb{R} \backslash\{0\}$.

Пусть $u(t)=\operatorname{tr} A_{1}(t), v(t)=\operatorname{tr} A_{2}(t)$. Так как функции $A_{2}(t), \boldsymbol{B}_{2}(t), C_{2}(t)$ имеют вид (12), для системы уравнений (7) рассмотрим задачу Коши

$$
\begin{gathered}
\dot{A}_{1}(t)=4 A_{1}(t) A_{2}(t)+\tau v(t) A_{1}(t)+\sigma u(t) A_{2}(t),\left.\quad A_{1}(t)\right|_{t=0}=A_{1}(0), \\
\dot{B}_{1}(t)=\left[2 A_{2}(t)+\tau v(t) I\right] \boldsymbol{B}_{1}(t)+\left[2 A_{1}(t)+\sigma u(t) I\right] \boldsymbol{B}_{2}(t),\left.\quad \boldsymbol{B}_{1}(t)\right|_{t=0}=\boldsymbol{B}_{1}(0), \\
\dot{C}_{1}(t)=\tau v(t) C_{1}(t)+\sigma u(t) C_{2}(t)+2\left(\boldsymbol{B}_{1}(t), \boldsymbol{B}_{2}(t)\right),\left.\quad C_{1}(t)\right|_{t=0}=C_{1}(0) .
\end{gathered}
$$

ТеОрема 6. Пусть $p=2$ и заданы вещественные симметричные матрицы $A_{1}(0), A_{2}(0) \in M_{n}(\mathbb{R})$ со свойством $A_{1}(0) A_{2}(0)=A_{2}(0) A_{1}(0)$, вектор-столбцы $\boldsymbol{B}_{1}(0), \boldsymbol{B}_{2}(0) \in M_{n, 1}(\mathbb{R})$, связанные соотношением $\boldsymbol{B}_{2}(0)=\frac{1}{\beta} S D(0) S^{\prime} \boldsymbol{B}_{1}(0)$, и скаляры $C_{1}(0), C_{2}(0) \in \mathbb{R}$, определяемые согласно

$$
C_{1}(0)=\frac{1}{2 \beta}\left|\boldsymbol{B}_{1}(0)\right|^{2}, \quad C_{2}(0)=\frac{1}{2 \beta^{2}}\left(S^{\prime} \boldsymbol{B}_{1}(0), D(0) S^{\prime} \boldsymbol{B}_{1}(0)\right)=\frac{1}{2 \beta}\left(\boldsymbol{B}_{1}(0), \boldsymbol{B}_{2}(0)\right) .
$$

Пусть, помимо этого, функиия $z(t)$ является вещественным решением задачи Kouи

$$
\dot{z}(t)=\sum_{k=1}^{m}\left[1-2 d_{k}(0) z(t)\right]^{2 /(m+2)}, \quad z(0)=0 .
$$

Тогда задача Коши (11), (14), нагруженная алгебраическими уравнениями (8), обладает вещественным решением

$$
\begin{gathered}
A_{2}(t)=\dot{z}(t) S Q(t) D(0) S^{\prime}=\dot{z}(t) S Q(t) S^{\prime} A_{2}(0), \quad \boldsymbol{B}_{1}(t)=[\dot{z}(t)]^{1 / 2} \boldsymbol{B}_{1}(0), \\
\boldsymbol{B}_{2}(t)=\frac{1}{\beta} \dot{z}(t) S Q(t) D(0) S^{\prime} \boldsymbol{B}_{1}(0)=\frac{1}{\beta} A_{2}(t) \boldsymbol{B}_{1}(0), \\
C_{2}(t)=\frac{1}{2 \beta^{2}} \dot{z}(t)\left(S Q(t) D(0) S^{\prime} \boldsymbol{B}_{1}(0), \boldsymbol{B}_{1}(0)\right)=\frac{1}{2 \beta^{2}}\left(A_{2}(t) \boldsymbol{B}_{1}(0), \boldsymbol{B}_{1}(0)\right), \\
A_{1}(t)=\beta[\dot{z}(t)]^{1 / 2} S E_{m} S^{\prime}=[\dot{z}(t)]^{1 / 2} A_{1}(0), \quad C_{1}(t)=\frac{1}{2 \beta}[\dot{z}(t)]^{1 / 2}\left|\boldsymbol{B}_{1}(0)\right|^{2} .
\end{gathered}
$$

Кроме того, $A_{1}(t), A_{2}(t)$ - вещественные симметричные матрицы для всех $t \in$ domain $A_{1}(t), t \in$ domain $A_{2}(t)$ соответственно. Здесь

$$
\begin{gathered}
Q(t)=\operatorname{diag}\left[\left[1-2 d_{1}(0) z(t)\right]^{-1}, \ldots,\left[1-2 d_{m}(0) z(t)\right]^{-1}, 1, \ldots, 1\right], \\
D(0)=\operatorname{diag}\left[d_{1}(0), \ldots, d_{m}(0), 0, \ldots, 0\right]
\end{gathered}
$$

$d_{l}(0) \in \mathbb{R} \backslash\{0\}$ - собственные значения матрицы $A_{2}(0), l=1,2, \ldots, m, \beta \in \mathbb{R} \backslash\{0\}$.

Объединяя утверждение 1 и теорему 6, заключаем, что справедлива

ТЕОРемА 7. Уравнение нелинейной диффузии

$$
u_{t}=\nabla \cdot\left(u^{-4 /(m+2)} \nabla u\right), \quad u \triangleq u(\boldsymbol{x}, t): \Omega \times \overline{\mathbb{R}}^{+} \rightarrow \mathbb{R}^{+}, \quad \boldsymbol{x} \in \mathbb{R}^{n},
$$

имеет точное неавтомодельное анизотропное по пространственным переменным явное неотрицательное решение вида (3) при $p=2 u \lambda=-4 /(m+2)$. При этом функиии $A_{k}(t), \boldsymbol{B}_{k}(t), C_{k}(t)$ определяются формулами $(15)$, где $m \in\{1, \ldots, n\}$, $k=1,2$. 
ЗАмечАниЕ 1. Групповая классификация уравнения (16) при $m=n$ проведена в работе [18]. В этом исследовании отмечено, что при любом $n \in \mathbb{N}$ и $K(u)=u^{-4 /(n+2)}$ происходит значительное расширение допустимой группы преобразований для уравнения (16). При этом особо выделяется двумерный случай: $n=2, K(u)=1 / u$, когда группа допустимых преобразований бесконечномерна.

Наконец, поясним смысл алгебраических соотношений

$$
\left(I-E_{m}\right) S^{\prime} B_{k}(0)=0, \quad k=1,2 .
$$

С этой целью перепишем (17) в виде $\boldsymbol{B}_{k}(0)=S E_{m} S^{\prime} \boldsymbol{B}_{k}(0)$, где $S \in M_{n}(\mathbb{R})$ - вешественная ортогональная матрица, $\boldsymbol{B}_{k}(0) \in M_{n, 1}(\mathbb{R})$ - вектор-столбцы, $E_{m}=\operatorname{diag}[1, \ldots, 1$, $0, \ldots, 0] \in M_{n}(\mathbb{R})$ - матрица, в которой число единиц на диагонали равно $m \in\{1, \ldots, n\}$. Далее введем обозначение $P_{m}=S E_{m} S^{\prime}$, где $S, S^{\prime}$ - невырожденные (неособые) матрицы. Тем самым, $\boldsymbol{B}_{k}(0)=P_{m} \boldsymbol{B}_{k}(0), P_{m}^{2}=P_{m}$, т.е. $P_{m} \in M_{n}(\mathbb{R})$ - вещественная симметричная идемпотентная матрица. Причем $P_{m}$ всегда можно привести к диагональному виду и, кроме того, $\operatorname{tr} P_{m}=m$. Помимо этого, хорошо известно, что вещественная симметричная матрица $P_{m}$ ранга $m$ является идемпотентной тогда и только тогда, когда $m$ ее собственных значений равны 1 , а остальные $n-m$ равны 0 . Более того, согласно [22] евклидово пространство $\mathbb{R}^{n}$ представимо в виде прямой суммы подпространств: $\mathbb{R}^{n}=L_{0} \oplus L_{1}$, где $L_{0}=\operatorname{range} P_{m}, L_{1}=\operatorname{ker} P_{m}$ - область значений и ядро матрищы $P_{m}$ соответственно. Очевидно, что векторы $\boldsymbol{B}_{k}(0) \in L_{0}$, где $k=1,2$.

С другой стороны, вводя в рассмотрение вектор-столбцы $\widehat{\boldsymbol{B}}_{k}(0)=E_{m} S^{\prime} \boldsymbol{B}_{k}(0)$ получим, что $\boldsymbol{B}_{k}(0)=S \widehat{\boldsymbol{B}}_{k}(0)$. Ясно, что $\widehat{\boldsymbol{B}}_{k}(0)$ принадлежит множеству $\mathscr{L}_{m}=\left\{x \in \mathbb{R}^{n}\right.$ : $\left.x_{m+1}=\cdots=x_{n}=0\right\}$.

Итак, для каждого фиксированного $k$ алгебраическое соотношение (17) вьполняется тогда и только тогда, когда существует вектор-столбец $\widehat{\boldsymbol{B}}_{k}(0) \in \mathscr{L}_{m}$ такой, что $\boldsymbol{B}_{k}(0)=S \widehat{\boldsymbol{B}}_{k}(0)$.

ПРИМЕР 1. Пусть $n=3, d_{l}=d_{l}(0) \in \mathbb{R}^{+}, l=1,2,3$. Тогда при $\lambda=-1$ решение задачи Коши (10) выражается в эллиптических функциях Якоби [23]. Например, приведем одно из восьми вещественных решений, найденньх авторами. Итак, если $d_{3}>d_{2}>d_{1}>0$, то

$$
z(t)=\frac{d_{2}-d_{3} \operatorname{sn}^{2}\left(\sqrt{d_{2}\left(d_{3}-d_{1}\right)} t+\mathrm{sn}^{-1}\left(\sqrt{d_{2} / d_{3}}, k\right), k\right)}{2 d_{2} d_{3} \mathrm{cn}^{2}\left(\sqrt{d_{2}\left(d_{3}-d_{1}\right)} t+\mathrm{sn}^{-1}\left(\sqrt{d_{2} / d_{3}}, k\right), k\right)},
$$

где $\operatorname{sn}(\cdot, k), \operatorname{cn}(\cdot, k)$ - эллиптические синус и косинус Якоби с модулем

$$
k=\sqrt{\frac{d_{3}\left(d_{2}-d_{1}\right)}{d_{2}\left(d_{3}-d_{1}\right)}}, \quad 0<k<1 ;
$$

$\mathrm{sn}^{-1}(\cdot, k)$ - функция обратная к $\operatorname{sn}(\cdot, k)$. Если $d_{1}=d_{2}<d_{3}$, то $k=0$ и

$$
z(t)=\frac{d_{2}-d_{3} \sin ^{2}\left(\sqrt{d_{2}\left(d_{3}-d_{2}\right)} t+\arcsin \sqrt{d_{2} / d_{3}}\right)}{2 d_{2} d_{3} \cos ^{2}\left(\sqrt{d_{2}\left(d_{3}-d_{2}\right)} t+\arcsin \sqrt{d_{2} / d_{3}}\right)} .
$$

Если $d_{3}=d_{2}>d_{1}$, то $k=1$ и

$$
z(t)=\frac{d_{1}-d_{2} \operatorname{sech}^{2}\left(\sqrt{d_{2}\left(d_{2}-d_{1}\right)} t+\operatorname{arcth} \sqrt{\left(d_{2}-d_{1}\right) / d_{2}}\right)}{2 d_{1} d_{2} \operatorname{th}^{2}\left(\sqrt{d_{2}\left(d_{2}-d_{1}\right)} t+\operatorname{arcth} \sqrt{\left(d_{2}-d_{1}\right) / d_{2}}\right)} .
$$




\section{СПИСОК ЦИТИРОВАННОЙ ЛИТЕРАТУРЫ}

[1] Кершнер Р.О некоторых свойствах обобщенных решений квазилинейных вырождающихся параболических уравнений // Acta Math. Hungar. 1978. V. 32. № 3-4. P. 301-330.

[2] Галактионов В. А., Дородницын В. А., Еленин Г. Г., Курдюмов С. П., Самарский А. А. Квазилинейное уравнение теплопроводности: обострение, локализация, симметрия, точные решения, асимптотики, структуры // Итоги науки и техн. Соврем. пробл. матем. Новейшие достижения. Т. 28. М.: ВИНИТИ, 1987. С. 95-205.

[3] Самарский А. А., Галактионов В. А., Курдюмов С. П., Михайлов А. П. Режимы с обострением в задачах для квазилинейных параболических уравнений. М.: Наука, 1987.

[4] Галактионов В. А., Посашков С. А. О новых точных решениях параболических уравнений с квадратичными нелинейностями // ЖВМиМФ. 1989. Т. 29. № 4. С. 497-506..

[5] Galaktionov V. A. On new exact blow-up solutions for nonlinear heat conduction equations with source and applications // Differential Integral Equations. 1990. V. 3. № 5. P. 863-874.

[6] Galaktionov V.A. Invariant subspaces and new explicit solution to evolution equations with quadratic nonlinearities. School of Math. Report no. AM-91-11: Univ. Bristol, 1991.

[7] Galaktionov V.A. Invariant subspaces and new explicit solution to evolution equations with quadratic nonlinearities // Proc. Roy. Soc. Edinburgh Sect. A. 1995. V. 125. P. 225-246.

[8] King J. R. Exact multidimensional solutions to some nonlinear diffusion equations // Quart. J. Mech. Appl. Math. 1993. V. 46. № 3. P. 419-436.

[9] Пухначев В. В. Многомерные точные решения уравнения нелинейной диффузии // Прикл. механика и технич. физика. 1995. Т. 36. № 2. С. 23-31.

[10] Meirmanov A. M., Pukhnachev V. V., Shmarev S. I. Evolution Equations and Lagrangian Coordinates. Berlin-New York: de Gruyter, 1997.

[11] Косыгина Е. Р. Об анизотропных точных решениях многомерного уравнения нестационарной фильтрации // ЖВМиМФ. 1995. Т. 35. №2. С. 241-259.

[12] Рудых Г. А., Семёнов Э.И. Новые точные решения одномерного уравнения нелинейной диффузии // Сиб. матем. ж. 1997. Т. 38. № 5. С. 1130-1139.

[13] Рудых Г. А., Семёнов Э. И. О новых точных решениях одномерного уравнения нелинейной диффузии с источником (стоком) // ЖВМиМФ. 1998. Т. 38. №6. С. 971-977.

[14] Калашников А. С. Некоторые вопросы качественной теории нелинейных вырождающихся параболических уравнений второго порядка // УМН. 1987. Т. 42. № 2. С. 135-176.

[15] Рудых Г. А., Семёнов Э. И. Точные неотрицательные решения многомерного уравнения нелинейной диффузии // Сиб. матем. ж. 1998. Т. 39. № 5. С. 1129-1138.

[16] Рудых Г. А., Семёнов Э. И. Точные неавтомодельные решения уравнения $u_{t}=\Delta \ln u$ // Матем. заметки. (в печати).

[17] Рудых Г. А., Семёнов Э. И. Точные решения многомерного уравнения нелинейной диффузии // Изв. РАН. Сер. матем.. (в печати).

[18] Галактионов В.А., Посашков С. А., Свирщевский С. Р. Обобщенное разделение переменных для дифференциальных уравнений с полиномиальными нелинейностями // Дифференц. уравнения. 1995. Т. 31. № 2. С. 253-261.

[19] Рождественский Б. Л., Яненко Н.Н. Системы квазилинейных уравнений. М.: Наука, 1978.

[20] Овсянников Л. В. Групповой анализ дифференциальных уравнений. М.: Наука, 1978.

[21] Ибрагимов Н. Х. Группы преобразований в математической физике. М.: Наука, 1983.

[22] Хорн Р., Джонсон Ч. Матричный анализ. М.: Мир, 1989.

[23] Ахиезер Н. И. Элементы теории эллиптических функций. М.: Гостехиздат, 1948.

Институт динамики систем и теории управления СО РАН 